\title{
Potential cardiovascular consequences of switching from atorvastatin to generic simvastatin in the Netherlands
}

\author{
D. Liew $\cdot$ K. Webb $\cdot$ W.-J. Meerding $\cdot$ E. Buskens $・$ \\ J. W. Jukema
}

Published online: 10 January 2012

(C) Springer Media / Bohn Stafleu van Loghum 2012

\begin{abstract}
Background The statin authorisation form implemented in the Netherlands in January 2009 has led to significant switching of patients from atorvastatin to generic simvastatin, but often to less than equipotent doses. We sought to assess the potential consequences of this.

Methods A modelling analysis was undertaken using data from a pharmacy database covering the majority of drug prescriptions in the Netherlands. Recent meta-analyses provided data on the dose-specific, lipid-modifying potencies of atorvastatin and simvastatin, and the relationship between
\end{abstract}

Previous presentations This work was presented at the ESC 2010 Congress (28 August to 1 September 2010, Stockholm), as oral abstract No. 3562 .

Funding This work was supported by Pfizer Limited.

D. Liew $(\bowtie)$

Melbourne EpiCentre, Department of Medicine (RMH),

University of Melbourne,

7East, The Royal Melbourne Hospital,

Parkville, VIC 3050, Australia

e-mail: danny.liew@mh.org.au

K. Webb

Sydney, Australia

W.-J. Meerding

Interhealth B.V.,

Dordrecht, the Netherlands

E. Buskens

University Medical Center Groningen,

Groningen, the Netherlands

J. W. Jukema

Leids Universitair Medisch Centrum,

Leiden, the Netherlands reduction in low-density lipoprotein cholesterol (LDL-C) achieved by statin therapy and relative reduction in risk of cardiovascular disease (CVD).

Results In the first quarter of 2009, 33.7\%, 47.2\% and $19.1 \%$ of Dutch patients initially on atorvastatin were switched to less potent, equipotent and more potent doses of simvastatin, respectively. The net effect was estimated to be a $6.8 \%$ increase in LDL-C. Assuming a pre-switch LDL$\mathrm{C}$ of $2 \mathrm{mmol} / \mathrm{L}$, the predicted relative increases $(95 \% \mathrm{CI})$ in the risks of all-cause mortality and major cardiovascular events were $1.7 \%(0.9 \%-2.6 \%)$ and $2.8 \%(1.6 \%-4.1 \%)$, respectively.

Conclusions In the Netherlands, policy-driven switching from atorvastatin to generic simvastatin led overall to less potent doses being used, with possible significant clinical implications.

Keywords HMG-CoA reductase inhibitors (statins) · Health policy · Drug switching $\cdot$ Cardiovascular diseases

\section{Introduction}

Confronted with very large and ever-increasing healthcare budgets, healthcare payers in many countries have sought to restrain pharmaceutical costs, including through a variety of policy-driven initiatives such as generic prescribing metrics (UK and Netherlands), restrictions to expensive drugs (Australia) and population-based switching policies (Norway).

HMG CoA reductase inhibitors (statins), which are used to modify serum lipid levels and reduce the risk of cardiovascular disease (CVD), are one class of medicines in which 'containment' policies have been implemented because simvastatin and pravastatin are now generic and available at much lower prices. Whereas CVD management guidelines 
issued by the US and European authorities [1, 2] do not recommend specific statins, some nationally issued guidelines do. For example, the National Institute for Therapeutics and Clinical Excellence (NICE) in the UK recommends starting therapy with simvastatin (40 $\mathrm{mg}$ daily)[3], while simvastatin $40 \mathrm{mg}$ and pravastatin $40 \mathrm{mg}$ are recommended in the current Dutch Cardiovascular Risk Management Guidelines[4]. In the Netherlands, only in situations where patients at very high risk of CVD (for example, recurrent myocardial infarction, familial predisposition and multiple risk factors), or where the preferred statins would not achieve the recommended goal of $<2.5 \mathrm{mmol} / 1$ for low-density lipoprotein cholesterol (LDLC), do the guidelines recommend more potent, still-branded statins (atorvastatin or rosuvastatin).

Following policy advice that was directed at controlling the cost of statins[5], the Dutch Ministry of Health revised reimbursement conditions for statins in line with the Dutch Cardiovascular Risk Management Guidelines in January 2009. Health insurers then introduced an authorisation form for atorvastatin, rosuvastatin, fluvastatin and ezetimibe, which required clinicians to state that these drugs were being prescribed in accordance with the Guidelines. As intended, the authorisation process led to many Dutch patients being switched from branded statins to generic statins[6]. For example, in the first 3 months of 2009, respectively, $14.8 \%, 15.6 \%$ and $14.5 \%$ of patients initially on atorvastatin were switched to generic simvastatin. However, many of these switches were to less potent doses in terms of LDL-C lowering.

We sought to estimate the potential impact, in terms of LDL-C control and subsequent cardiovascular risk, of patterns of atorvastatin-to-simvastatin switching recently observed in the Netherlands.

\section{Methods}

Patterns of switching from atorvastatin to generic simvastatin

Data regarding statin switching patterns were derived from the Dutch Foundation for Pharmaceutical Statistics (Stichting Farmaceutische Kengetallen, SFK)[6]. The SFK directly gathers prescribing data from over 1800 (of the nearly 2000) community pharmacies from across the Netherlands, which collectively serve 15 million (90\%) of the Dutch population.

Statin switching data were drawn from the period January to March 2009, immediately after the implementation of the statin authorisation form. In this period, 39,031 Dutch patients were switched from atorvastatin to generic simvastatin, representing $15 \%$ of the patients previously taking atorvastatin. Dose-specific switching patterns from atorvastatin to generic simvastatin for these 39,031 patients are summarised in Table 1.

LDL-C lowering effects of switching from atorvastatin and generic simvastatin

The proportional LDL-C lowering effects of atorvastatin and simvastatin, at various daily doses, were derived from the meta-analysis by Law et al.[7], and are summarised in Table 2. Using these data, proportional changes to LDL-C were calculated for each dose-specific switch from atorvastatin to generic simvastatin. For example, if a patient had been switched from $10 \mathrm{mg}$ daily of atorvastatin (associated with a $37.3 \%$ reduction in LDL-C) to $10 \mathrm{mg}$ daily of simvastatin (associated with a $27.3 \%$ reduction in LDL-C), then a $10 \%$ (37.3\% minus $27.3 \%)$ rise in LDL-C was assumed.

Cardiovascular risk effects of switching from atorvastatin and generic simvastatin

To estimate changes in cardiovascular risk associated with observed patterns of switching from atorvastatin to generic simvastatin, the proportional changes to LDL-C expected to arise from switching were combined with data from a metaanalysis undertaken by the Cholesterol Treatment Trialists Collaborators (CTTC)[8], which estimated relative risk reductions conferred by statin-induced reduction in LDL-C for various cardiovascular outcomes. A summary of the key results for the CTTC meta-analysis is provided in Table 3.

\section{Uncertainty analyses}

Uncertainty (probabilistic sensitivity) analysis was undertaken via Monte Carlo simulation[9] with 10,000 iterations, based on the following variations to key input parameters:

- LDL-C lowering effects of atorvastatin and simvastatin at the various daily doses: triangular uncertainty ranges capturing the lower limits of the $95 \%$ confidence

Table 1 Dose-specific switching from atorvastatin to generic simvastatin observed among 39,031 Dutch patients in the first 3 months of 2009[6]

Switched simvastatin daily dose (mg)

\begin{tabular}{llll}
\hline 0 & 20 & 40 & 80
\end{tabular}

Original atorvastatin daily dose $(\mathrm{mg})$

\begin{tabular}{llllll}
10 & 2006 & 6932 & 7417 & 13 & 16,368 \\
20 & 215 & 4042 & 11,034 & 27 & 15,318 \\
40 & 83 & 515 & 6290 & 457 & 7345 \\
& 2304 & 11,489 & 24,741 & 497 & 39,031 \\
\hline
\end{tabular}


Table 2 Dose-specific proportional LDL-C lowering effects $(95 \%$ confidence intervals) of atorvastatin and simvastatin[7]

\begin{tabular}{llll}
\hline & \multicolumn{2}{l}{ Atorvastatin } & Simvastatin \\
\hline Daily dose & $10 \mathrm{mg}$ & $37.3 \%(33.8 \%-41.0 \%)$ & $27.3 \%(25.4 \%-29.2 \%)$ \\
& $20 \mathrm{mg}$ & $43.1 \%(39.6 \%-46.9 \%)$ & $32.1 \%(30.4 \%-34.0 \%)$ \\
& $40 \mathrm{mg}$ & $49.2 \%(44.2 \%-54.0 \%)$ & $37.1 \%(34.6 \%-39.6 \%)$ \\
& $80 \mathrm{mg}$ & $55.0 \%(48.1 \%-61.7 \%)$ & $41.9 \%(38.1 \%-45.6 \%)$ \\
\hline
\end{tabular}

interval, the point estimates and the upper limits of the 95\% confidence interval (Table 2 )

- Relative risk reductions associated with every $\mathrm{mmol} / \mathrm{L}$ reduction in LDL-C conferred by statins: triangular uncertainty ranges capturing the lower limits of the $95 \%$ confidence interval, the point estimates and the upper limits of the $95 \%$ confidence interval (Table 3).

In brief, Monte Carlo simulation involves undertaking multiple repeated analyses, with each analysis sampling an input value from a specified uncertainty range (as with LDL-C lowering and relative risk reductions above). Multiple outputs are generated from the repeated analyses, which collectively reflect the uncertainty inherent in the inputs, and around which uncertainty ranges can be described.

All analyses were undertaken in Microsoft Excel (Microsoft Corporation, Redmond, WA, USA). Monte Carlo simulation was enabled in Microsoft Excel by addition of the software macro@Risk (Palisade Corporation, New York, NY, USA).

\section{Results}

Patterns of switching from atorvastatin and generic simvastatin

Assuming a 2:1 ratio of LDL-C lowering potency for atorvastatin versus simvastatin[7, 10], SFK data from the first

Table 3 Relative risks (95\% confidence intervals) associated with every $1 \mathrm{mmol} / \mathrm{L}$ reduction in LDL-C achieved with statins[8]

\begin{tabular}{ll}
\hline Outcome & Relative risk \\
\hline All-cause mortality & $0.88(0.84-0.91)$ \\
Coronary mortality & $0.81(0.76-0.85)$ \\
Myocardial infarction or coronary death & $0.77(0.74-0.80)$ \\
Coronary vascularisation $_{\text {Stroke (non-fatal and fatal) }}$ & $0.76(0.73-0.80)$ \\
Major cardiovascular event $^{\mathrm{a}}$ & $0.83(0.78-0.88)$ \\
\hline
\end{tabular}

${ }^{\text {a }}$ Composite of myocardial infarction or coronary death, coronary vascularisation and stroke
3 months of 2009 indicate that $33.7 \%, 47.2 \%$ and $19.1 \%$ of switches from atorvastatin were to less potent, equipotent and more potent doses, respectively, of generic simvastatin. The weighted average daily dose of initial atorvastatin was $19.6 \mathrm{mg}$, and $32.9 \mathrm{mg}$ for switched generic simvastatin.

LDL-C lowering effects of switching from atorvastatin and generic simvastatin

Based on data by Law et al.[7] regarding the proportional LDL-C lowering effects of atorvastatin and simvastatin (Table 2), expected proportional changes to LDL-C were calculated for each dose-specific switch from atorvastatin to generic simvastatin, and are summarised in Table 4 . Combining these data with the patterns of switching observed in the Netherlands in the first 3 months of 2009 (Table 1), the predicted net effect was a $6.8 \%$ increase in LDL-C.

Cardiovascular risk effects of switching from atorvastatin and generic simvastatin

The predicted relative changes in risk for various cardiovascular outcomes were dependent on the baseline (atorvastatin-treated, pre-switch) LDL-C levels. Weighted-average results for five assumed levels of baseline LDL-C (2.0, 2.5, 3.0, 3.5 and $4.0 \mathrm{mmol} / \mathrm{L}$ ) are summarised in Table 5 .

\section{Discussion}

Implementation of the statin authorisation form in the Netherlands in January 2009 resulted in many patients being switched from branded atorvastatin to generic simvastatin at less than therapeutically equivalent doses.

Based on the observed switching patterns, our simple analysis predicts an increase in cardiovascular events that have to be weighed against the net cost savings from switching. It highlights the need for health policy makers to be conscious of the potential adverse, unintended consequences of policy

Table 4 Expected proportional increase in LDL-C with dose-specific switching from atorvastatin to simvastatin (combination of data from Tables 1 and 2)

\begin{tabular}{|c|c|c|c|c|}
\hline & \multicolumn{4}{|c|}{ Switched simvastatin daily dose (mg) } \\
\hline & 10 & 20 & 40 & 80 \\
\hline \multicolumn{5}{|c|}{ Original atorvastatin daily dose (mg) } \\
\hline 10 & $10.0 \%$ & $5.2 \%$ & $0.2 \%$ & $-4.6 \%$ \\
\hline 20 & $15.8 \%$ & $11.0 \%$ & $6.0 \%$ & $1.3 \%$ \\
\hline 40 & $21.9 \%$ & $17.1 \%$ & $12.1 \%$ & $7.3 \%$ \\
\hline
\end{tabular}


Table 5 Predicted relative changes in risk (95\% confidence intervals) for various outcomes, according to five pre-switch (atorvastatin-treated) LDL-C levels

\begin{tabular}{|c|c|c|c|c|c|}
\hline & \multicolumn{5}{|c|}{ Pre-switch (atorvastatin-treated) LDL-C (mmol/L) } \\
\hline & 2.0 & 2.5 & 3.0 & 3.5 & 4.0 \\
\hline All-cause mortality & $1.7 \%(0.9-2.6)$ & $2.1 \%(1.1-3.3)$ & $2.5 \%(1.3-3.9)$ & $2.9 \%(1.5-4.6)$ & $3.3 \%(1.8-5.2)$ \\
\hline Coronary mortality & $2.6 \%(1.4-4.0)$ & $3.3 \%(1.8-5.0)$ & $3.9 \%(2.1-6.0)$ & $4.6 \%(2.5-7.0)$ & $5.2 \%(2.8-8.0)$ \\
\hline MI or coronary death & $3.1 \%(1.7-4.5)$ & $3.9 \%(2.2-5.7)$ & $4.7 \%(2.6-6.8)$ & $5.4 \%(3.0-8.0)$ & $6.2 \%(3.5-9.1)$ \\
\hline Coronary vascularisation & $3.2 \%(1.8-4.7)$ & $4.0 \%(2.2-5.9)$ & $4.8 \%(2.6-7.0)$ & $5.6 \%(3.1-8.3)$ & $6.4 \%(3.6-9.3)$ \\
\hline Stroke (non-fatal and fatal) & $2.3 \%(1.2-3.6)$ & $2.9 \%(1.5-4.5)$ & $3.4 \%(1.8-5.4)$ & $4.0 \%(2.1-6.4)$ & $4.6 \%(2.4-7.2)$ \\
\hline Major cardiovascular event ${ }^{\mathrm{a}}$ & $2.8 \%(1.6-4.1)$ & $3.5 \%(2.0-5.2)$ & $4.2 \%(2.4-6.2)$ & $5.0 \%(2.8-7.2)$ & $5.7 \%(3.2-8.2)$ \\
\hline
\end{tabular}

$L D L-C$ low-density lipoprotein cholesterol. MI myocardial infarction

${ }^{a}$ Composite of myocardial infarction or coronary death, coronary vascularisation and stroke

changes, and for clinicians to be aware of dose equivalences among statins.

Observational data show that patients in other countries have also been switched to a less than therapeutically equivalent dose of a generic statin. In the US, Hess et al.[11] undertook a large retrospective analysis of 453,409 patients and found that of those who switched to simvastatin, $38 \%$ received a lower therapeutic dose. Similar proportions were noted by Aronow et al.[12]. In the Hess study, which reported on dose-specific switching patterns, the proportion of patients receiving a lower than equivalent dose of simvastatin ranged from $18 \%$ in those who had previously received $10 \mathrm{mg}$ atorvastatin to $73 \%$ in those who had previously received $40 \mathrm{mg}$ atorvastatin. This suggests that high-risk patients, who require more intensive therapy to reduce cardiovascular risk, are particularly prone to under-treatment as a result of switching to a less potent generic statin.

A retrospective study conducted in Germany found that $44.5 \%$ of 805 patients switched from atorvastatin received a dose of another statin in replacement that was less than therapeutically equivalent to their previous dose[13].

Similar to our findings, a recent US study by Rublee and Burke[14] noted that among 1048 patients who were switched from atorvastatin to simvastatin, switching was from a mean daily dose of $20.9 \mathrm{mg}( \pm 15.3)$ to a mean daily dose of $32.6 \mathrm{mg}( \pm 17.9)$.

The findings of our and others' studies suggest that many clinicians are unaware of the differences in potencies between the statins. This notion is supported by a US study which found that while $98.2 \%$ of patients who were switched to a generic version of their originally prescribed branded statin ('generic substitution') were prescribed the same dose, only $65.4 \%$ of patients switched to a different generic statin ('therapeutic substitution') received an equipotent dose[15]. Moreover, these percentages were estimated based on a therapeutic equivalence between atorvastatin and simvastatin of $1: 2$, rather than the more commonly accepted equivalence of between 1:3 and 1:4.
Our model estimates an increase in cardiovascular events based on the expected negative effect on LDL-C levels. This clinically intuitive assumption is supported by a number of observational studies from across the world showing that switching from atorvastatin to simvastatin is associated with a negative impact on LDL-C levels. A German study showed that patients who discontinued treatment with atorvastatin (ceased or switched to other lipid-lowering treatment) experienced significant increases in total cholesterol, LDL-C and triglyceride levels $(p<0.001)[13]$. In those who remained on atorvastatin, lipid levels did not change. The study by Aronow et al. in the US[12], which was based on patients at high cardiovascular risk (with a history of coronary heart disease or equivalent), found that switching from atorvastatin to simvastatin was associated with increased LDL-C levels (2.26 versus $2.36 \mathrm{mmol} / \mathrm{L} ; p=0.009)$. In the sub-group of patients who were prescribed a less potent dose of simvastatin, the difference in LDL-C was even higher (2.26 versus $2.46 \mathrm{mmol} / \mathrm{L} ; p=0.002)$.

We are aware of only two studies to date that have assessed the impact of switching in terms of cardiovascular outcomes, the first in the UK in a population treated in the community (comprising a mix of primary and secondary prevention patients)[16] and the second in Italy in patients with acute coronary syndromes (ACS)[17]. The UK study of 11,520 patients showed that those who switched from atorvastatin to simvastatin (2511 patients), compared with those who remained on atorvastatin (9009 patients), had a significantly increased adjusted risk of the composite endpoint of all-cause death and major cardiovascular event $(+30 \% ; p=0.03)$, a major cardiovascular event alone $(+43 \% ; p=0.008)$, and stroke $(+114 \% ; p=0.009)[16]$. Colivicchi et al. studied Italian ACS patients discharged on high-dose atorvastatin ( $80 \mathrm{mg}$ ) and found a statistically significant increased risk of adverse outcomes (all-cause death, non-fatal myocardial infarction and stroke) at 1 year $(p=0.004)$ among patients who were switched from 
atorvastatin $80 \mathrm{mg}$ to another statin therapy (including simvastatin, pravastatin, fluvastatin or a lower dose of atorvastatin)[17].

\section{Limitations}

The first limitation to our study is one common to all modelling analyses: use of data from a variety of sources (not all 'controlled trials') to extrapolate and suggest an association between changes in prescribing behaviour and cardiovascular outcomes. This introduces uncertainty that we attempted to capture via Monte Carlo simulation. Secondly, the switching data from the SFK, upon which this analysis was based, were obtained retrospectively and hence the reasons for switching were not known. It may be that that some patients were switched because of their being at lower cardiovascular risk. Regardless, that they were switched to less potent doses of simvastatin would still have led to increases in LDL-C levels and cardiovascular risk. Lastly, while the Dutch SFK data covered national prescribing practices, there are regional differences in the Netherlands that the study was unable to account for. As with all such analyses, the patient sample may not have been a representative population. If selection bias was present, we are not aware of the direction in which the results would have been biased.

\section{Conclusion}

In the Netherlands, policy-driven switching from atorvastatin to generic simvastatin led overall to less potent doses being used, with implications for increased risk of downstream CVD and death. Health policy makers need to be conscious of the potential adverse health consequences of financially motivated policy changes, and clinicians need to be aware of dose equivalences among statins.

\section{References}

1. Grundy SM, Cleeman JI, Merz CN, et al. Implications of recent clinical trials for the National Cholesterol Education Program Adult Treatment Panel III guidelines. Circulation. 2004;110:227-39.
2. Graham I, Atar D, Borch-Johnsen K, et al. European guidelines on cardiovascular disease prevention in clinical practice: executive summary: Fourth Joint Task Force of the European Society of Cardiology and Other Societies on Cardiovascular Disease Prevention in Clinical Practice (constituted by representatives of nine societies and by invited experts). Eur Heart J. 2007;28:2375-414.

3. National Institute for Health and Clinical Excellence. Statins for the prevention of cardiovascular events. Technology appraisal 94. 2008. Available at: http://www.nice.org.uk/nicemedia/live/11564/ 33151/33151.pdf

4. Kwaliteitsinstituut voor de gezondheidszorg CBO en Nederlands Huisartsen Genootschap Multidisciplinaire richtijn Cardiovasculair risicomanagement. (In Dutch.) Utrecht, 2006.

5. College voor Zorgverzekeringen CVZ, Pakketadvies 2008. (In Dutch.) Diemen, 2008.

6. Stichting Farmaceutische Kengetallen. Beoogde besparing op statines onzeker. Pharmaceutisch Weekblad. 2009;144. Available at: http://www.sfk.nl/

7. Law MR, Wald NJ, Rudnicka AR. Quantifying effect of statins on low density lipoprotein cholesterol, ischaemic heart disease, and stroke: systematic review and meta-analysis. BMJ. 2003;326:1423.

8. Baigent C, Keech A, Kearney PM, et al. Efficacy and safety of cholesterol-lowering treatment: prospective meta-analysis of data from 90,056 participants in 14 randomised trials of statins. Lancet. 2005;366:1267-78.

9. Briggs AH. Handling uncertainty in cost-effectiveness models. PharmacoEconomics. 2000;17:479-500.

10. Rogers SL, Magliano DJ, Levison DB, et al. A dose-specific metaanalysis of lipid changes in randomized controlled trials of atorvastatin and simvastatin. Clin Ther. 2007;29:242-52.

11. Hess G, Sanders KN, Hill J, et al. Therapeutic dose assessment of patient switching from atorvastatin to simvastatin. Am J Manag Care. 2007;13 Suppl 3:S80-5.

12. Aronow HD, Hess G, Hill J, et al. Switching from atorvastatin to simvastatin in patients at high cardiovascular risk: effects on lowdensity lipoprotein cholesterol. Am J Ther. 2010;17:167-75.

13. Bestehorn K, Greiner W. Effects of the introduction of fixed prices for statins on lipid levels in Germany. J Publ Health. 2008;16:2159.

14. Rublee DA, Burke JP. LDL-C goal attainment in patients who remain on atorvastatin or switch to equivalent or non-equivalent doses of simvastatin: a retrospective matched cohort study in clinical practice. Postgrad Med. 2010;122:16-24.

15. Chapman RH, Benner JS, Girase P, et al. Generic and therapeutic statin switches and disruptions in therapy. Curr Med Res Opin. 2009;25:1247-60.

16. Phillips B, Roberts C, Rudolph AE, et al. Switching statins: the impact on patient outcomes. Br J Cardiol. 2007;14:280-5.

17. Colivicchi F, Tubaro M, Santini M. Clinical implications of switching from intensive to moderate statin therapy after acute coronary syndromes. Int J Cardiol. 2010. 